\title{
Improvement of Bonding-box Algorithm for Wireless Sensor Networks
}

\author{
Yedong $\mathrm{Xu}^{*}$, Caixia Liu ${ }^{*}$ and Xumin Sun \\ College of Computer Science, Inner Mongolia University, Huhehot 010021, China \\ ${ }^{*}$ Corresponding author
}

\begin{abstract}
The localization is an important technology for the sensor network. In this paper, we improved the large error of the Bonding-box algorithm in the case of the two anchor nodes and the disadvantages of the indistinguishable localization of coordinates. The algorithm is combined with the ranging technology RSSI, and is simple to be achieved without additional hardware equipment. It is found during the experiment that it has low accuracy of localization at the edge of sensor network on the situation with a small number of anchor nodes (mainly has two anchor nodes). And all the nodes in the range are located in the center of the range, which results in a low degree of identification. In this paper, we propose a new algorithm to reduce the error and improve the identifiable degree. MATLAB simulation is used to show that the algorithm is better than the Bonding-box algorithm which is based on the weighted centroid, and it can be applied to all of the current improved algorithms which are based on Bonding-box algorithm. And this algorithm could reduce the error of the improved algorithm.
\end{abstract}

Keywords-centroid algorithm; wireless sensor network; localization algorithm; bonding-box; RSSI

\section{INTRODUCTION}

In the Bounding-box approach [1], it uses the square overlapping area to locate the coordinates of unknown nodes. And this algorithm has following merits, such as it is simple to compute, convenient for use, without additional hardware, with a low cost, and a low power consumption, which is very suitable for wireless sensor networks localization [1]. Around the unknown nodes anchor, nodes are more intensive, and calculation of the locating error is less. But due to wireless sensor networks subject to cost constraints, and adding anchor node will greatly increase the network overhead and increase the power consumption, thus increasing the anchor node is unrealistic [2].Over this problem, many researchers have improved the Bonding-box algorithm [3]. A weighted centroid algorithm based on RSSI is proposed by Veike Chen and others [4], but this algorithm requires at least three anchor nodes to locate, and does not deal with the cases of two anchor nodes. In paper [5], three point and three point weighted centroid Bonding-box algorithm was proposed, however, it also needs three anchor nodes. In paper [6], a new weighted Bonding-box algorithm was proposed, to further improve the locating accuracy, but it requires four anchor nodes to operate. The paper [7] proposed a method to determine the area of the node by using the method of boundary delimitation and it uses the full search method to find the best estimating point. But the calculation of the algorithm is too large and it is not very suitable for wireless sensor networks [8].Sanabria-Russo[9] and other people proposed a flexible locating method, which can choose different locating methods according to the applying requirements, but it did not fundamentally solve the problems existing in the algorithm itself. The improved algorithm proposed in the paper [10] can only be used to solve the problem of indoor NLOS localization, and the computational complexity is relatively high. Reference [11] proposed a method based on fundamentally differential correction. Although this method reduces the errors, it cannot be applied to differential correction, if there are only two anchor nodes and the distance between the two anchor nodes is beyond the communicating radius. So it does not solve the problem of only two anchor nodes. The high precision locating algorithm based on Bonding-box is proposed by the paper [12], which can further improve the positioning accuracy by Regional excluding method. However, there are some locating errors, and there are some unknown nodes located in one center of the region, which results in a low degree of identification.

The paper [13] proposed weighted centroid locating algorithm, which solved the two anchor points of the locating accuracy in some degree and improved the degree of identification. It has a good effect, but it is limited to improve the locating accuracy of two anchor points. In the later part this paper, we focus on the simulation and comparison to this algorithm. Due to the above algorithms are all the improved algorithms of Bonding-box algorithm and the two anchor nodes location problem is not solved, the algorithm in this paper is applied on the basis of basic Bonding-box algorithm, and all the above algorithms can further reduce the error and improve the degree of identification by using algorithm we proposed in the case of two anchor nodes. In this paper, the algorithm of the paper [13] and the algorithm proposed in this paper are compared in error and the degree of identifications and we achieved a better effect.

\section{THE PRINCIPLE OF LOCATING ALGORITHM AND ITS SHORTAGE}

Bonding-box algorithm is firstly proposed by the paper [1]. In FIGURE I, the solid circle is the unknown node, and the hollow circle is anchor node of the known coordinates. The circle, which regards the anchor node as the center of it, represents the accessibly communicating area of that anchor node. And the square is an external square of the circle. If an unknown node is in the accessibly communicating area of the anchor node, it must also within the external square area. 


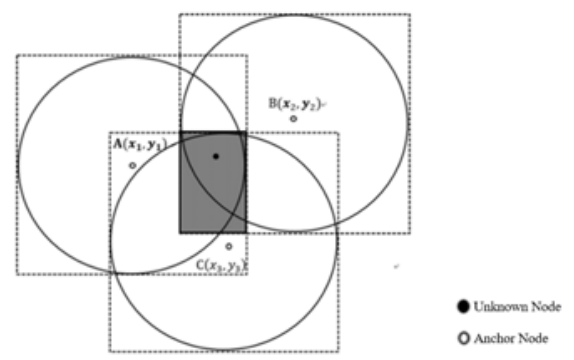

FIGURE I.BONDING-BOX ALGORITHM GRAPH

If the unknown node broadcasts a locating request message to the surrounding area, all the anchor nodes which the unknown node can reach will receive the message and then the anchor nodes broadcast their coordination's message to the surrounding. After receiving the messages, the unknown node will know that there are three anchors A, B, C within its communicating area. According to anchors A, B, C and communicating radius, the unknown node can get intersecting area of three rectangles. And the unknown node must be in the region of the shadowed region in Fig.1. Thus the coordinates of the unknown nodes can be replaced by calculating the center of the intersecting rectangle.

Bonding-box algorithm is simple and easy to be implemented, but the all nodes within the shadowed area can be defined as the center of the shadowed area. There are some big errors, these nodes' coordinate are same, and the degree of identification is very low. By the principle of Bounding-box locating algorithm, we can see the degree of the error depends on the number of the whole network's anchor nodes. If you want to more accurately locate an unknown node, it requires a minimum of three pieces of anchor locating information, and the only two anchor nodes will cause a very big error [14]. In order to reduce the error, it can be achieved by increasing the number of anchor nodes. But this will result in the increase of the cost and power consumption as well as the network traffic. Finally, it will lose the original advantages of the algorithm.

For this reason, many scholars mentioned above have put forward their improved algorithms. They made a very large improvement on the location of three or more anchor nodes around the unknown node. But as mentioned above, the above algorithms are generally not suitable for the case of only two anchor nodes around the unknown node. Therefore, this paper presented an improved Bounding-box algorithm to locate unknown nodes which has only two anchor nodes around. The algorithm can be applied in all the above improved algorithm based on Bonding-box to solve the large locating error in the above algorithms for the two anchor nodes.

\section{PRINCIPLE OF THE IMPROVED LOCATING ALGORITHM}

As is shown in FIGURE II(a), $x_{1}$ and $x_{2}$ were anchor nodes, and $\mathrm{A}, \mathrm{B}$ is unknown nodes. The two circles are communicating circles surrounding by $x_{1}$ and $x_{2}$. Draw two straight lines through the point $\mathrm{A}$ and $\mathrm{B}$, and the intersections are $A^{x}$ and $B^{s}$ respectively. Unknown nodes $\mathrm{A}$ and $\mathrm{B}$ are positioned at the center of the rectangular area ' $\mathrm{O}$ ' by using Bonding-box locating algorithm and the locating errors are AO and $\mathrm{BO}$ respectively, which is the hypotenuse of right-angled triangle. The algorithm of this paper is to use $A^{f}$ and $B^{\prime}$ to act as $A$ and $B$ locating coordinates, and the error of the localization is $A^{T} O$ and $B^{T} O$, which is a side of the right-angle triangle. From the triangle theorem we can know, each of the two sides is smaller than the hypotenuse. So it not only can reduce the locating error, distinguish the coordinates between $\mathrm{A}$ and $\mathrm{B}$, but also can improve the degree of identification despite there are other nodes in the region.

Then we will focus on how to get the coordinates of $\boldsymbol{A}^{r}$. We use the ranging technique RSSI to convert the received signal's attenuation of $x_{1}$ and $x_{2}$ into the distance. In this way, we can get the distance of A to $x_{1}$ and $x_{2}$, and we call it $d_{1}$ and $d_{2}$. Because the ranging technology RSSI is an inexpensive technology of distant measurement, and most of the wireless communicating module can inbuilt RSSI model. So it does not increase the cost of hardware. In FIGURE II(b) we know, if we taking $x_{1}$ and $x_{2}$ as the center of the circles and taking $d_{1}$ and $d_{2}$ as the radius of the circles, the intersections of the two circles will be $\mathrm{A}$ and $A^{E P}$. We can get the coordinates of $\mathrm{A}$ and $A^{T F}$ by solving the equation of the two circles. Then we take the center coordinates of the two intersections, which is what we need the position of $A^{r}$.

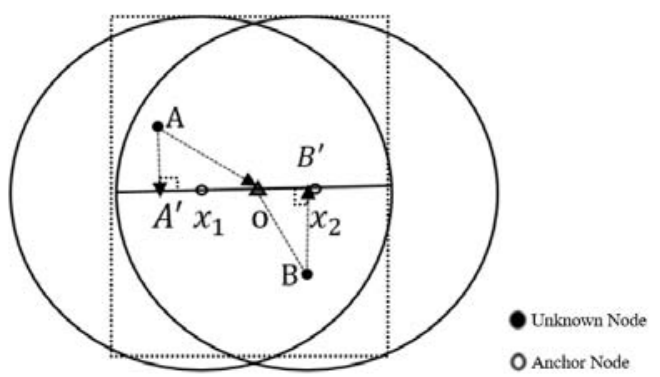

(a)

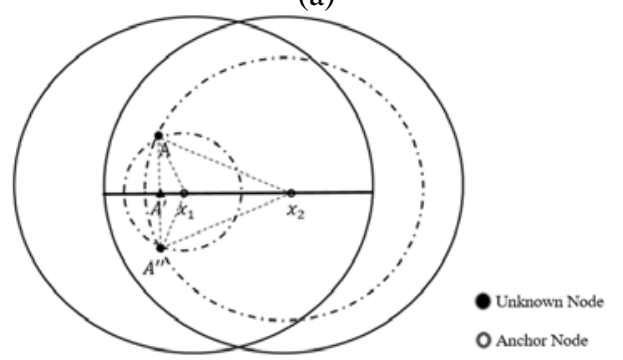

(b)

FIGURE II. IMPROVED BONDING-BOX ALGORITHM GRAPH

We call this improved algorithm as RB-box algorithm. Based on the above ideas and analysis, the achieving steps of RB-box algorithm are as follows:

Step 1: When the number of anchors of unknown nodes in a communicating area is not 2 , we can use the improved algorithm mentioned in the introduction to locate.

Step 2: When the number of anchors of unknown nodes in a communicating area is 2, we use RB-box algorithm to locate. 


\section{EXPERIMENTAL EVALUATION AND ANALYSIS}

Firstly, we compare the RB-box algorithm and the initial Bonding-box algorithm based on the simulation of experiment. We evaluate and analyze the RB-box algorithm. Secondly, we simulates and compares RB-box algorithm with the algorithm proposed by the paper [13]. In order to merely compare the performance of the locating accuracy and the recognizing accuracy of the whole wireless sensor network in the condition of two anchor nodes, we apply the initial Bonding-box algorithm to the whole simulating scenario, but in the case of the two anchor nodes, we use the algorithms proposed by the paper [13] and the RB-box algorithm respectively.

\section{A. Experiment Scene Layout}

Using MATLAB to build a $1000 * 1000$ rectangular area, we distribute 300 nodes randomly and evenly, which has 45 anchor nodes and 255 unknown nodes in the region of this area. The reason for this setting is that just to reach the point that all nodes are able to be located, so that the number of anchor nodes is minimum. Node distribution is shown in FIGURE III (a), where red * represent anchor nodes, and blue circles represent unknown nodes. FIGURE III(b) shows the relationship between the neighbor nodes, and the blue line between nodes indicates that the node can communicate with each node.

Because the wireless sensor nodes of the experiment are randomly distributed, the nodes distribution of each experiment and the neighbors are different. The FIGURE III(a) and FIGURE III(b) are the results of a randomly experiment, and they are a graph of node distribution and a graph of a neighbor relationship respectively.

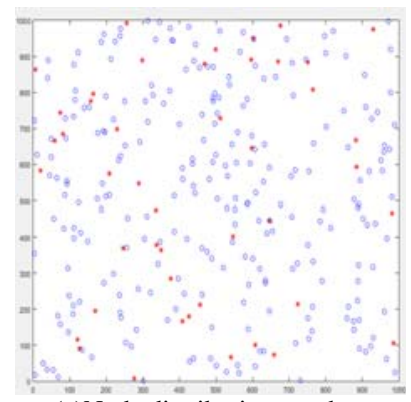

(a)Node distribution graph

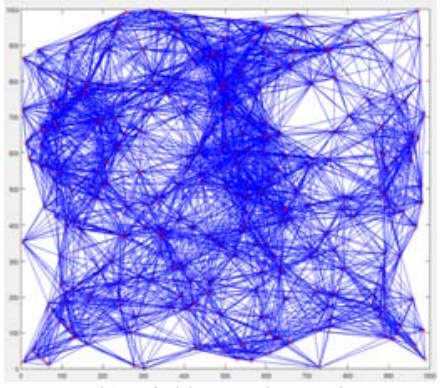

(b)Neighbor node graph
FIGURE III. NODE DEPLOYMENT GRAPH

\section{B. Experimental Evaluation and Analysis}

Through the MATLAB simulating experiment, we get the experimental results of FIGURE IV, FIGURE V and FIGURE VI, and the blue line represents the locating error. We put the places where the improvements are very obvious in circles. We can clearly see that the initial Bounding-box algorithm will locate all the nodes in one position and it has a very low degree of identification. Although the improved Bonding-box algorithm proposed by the paper [13] can roughly distinguish the location of the unknown nodes, the unknown nodes are still get together in one region and the degree of identification is relatively low. From the graph, we can see that the RB-box algorithm can distinguish the unknown nodes very well, and the degree of identification is higher.

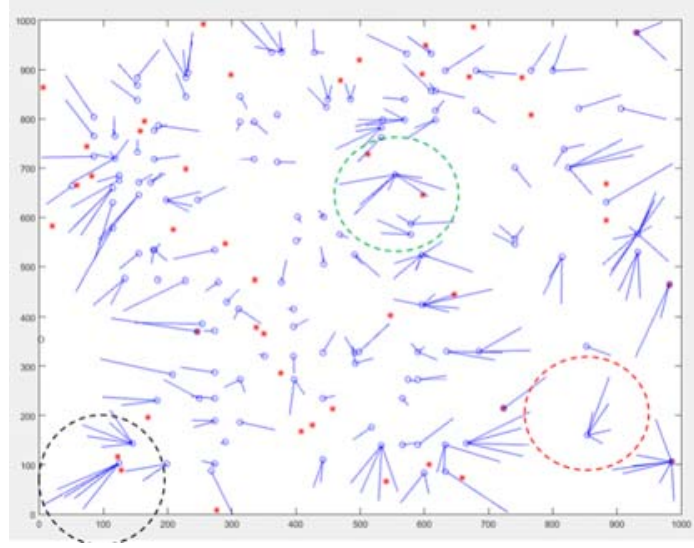

FIGURE IV. ERROR GRAPH OF INITIAL BONDING-BOX ALGORITHM

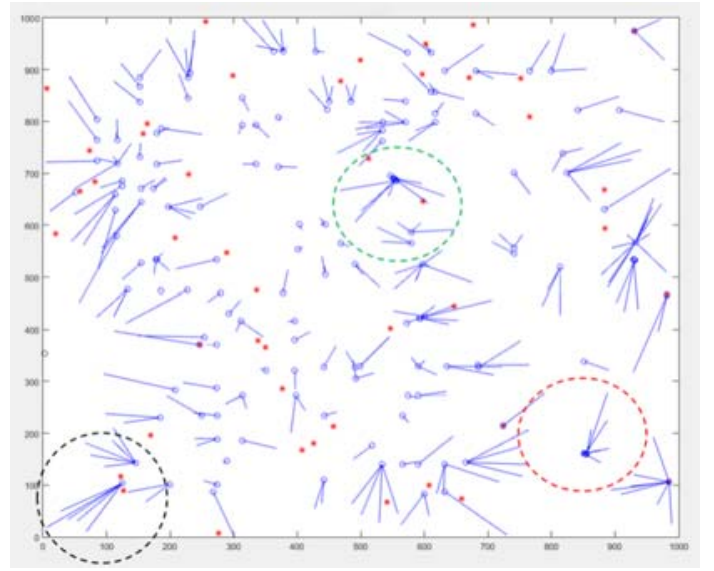

FIGURE V. ERROR GRAPH OF ALGORITHM PROPOSED BY PAPER [13]

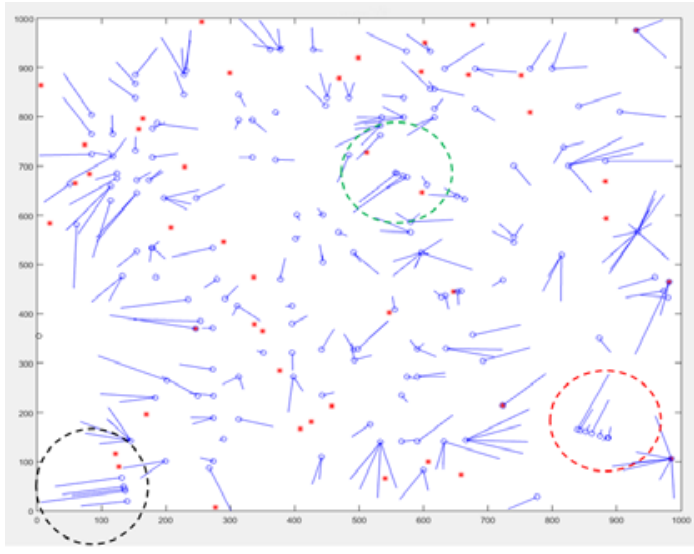

FIGURE VI. ERROR GRAPH OF RB-BOX ALGORITHM

Because the unknown nodes and anchor nodes are randomly distributed in the simulating experiment, the results of each experiment are different. In order to prove the performance of the algorithm proposed by our paper, we conducted 50 random experiments. Each experiment, we use three algorithms to locate. Then we will get 50 locating errors and calculate the average value of the error which was recorded 
in TABLE I. Because of the large number of experiments, the results of the 50 experiments are not listed all. FIGURE IV, FIGURE $\mathrm{V}$ and FIGURE VI is a random selection of the experimental results, but this is not the best one.

TABLE I. ERROR STATISTICS

\begin{tabular}{|c|c|c|c|}
\hline & $\begin{array}{c}\text { Error of initial } \\
\text { Bonding-box } \\
\text { algorithm }\end{array}$ & $\begin{array}{c}\text { Error of algorithm } \\
\text { proposed by paper } \\
{[13]}\end{array}$ & $\begin{array}{c}\text { Error of } \\
\text { RB-box } \\
\text { algorithm }\end{array}$ \\
\hline $\begin{array}{c}\text { The entire } \\
\text { network }\end{array}$ & $0.2849 \%$ & $0.2867 \%$ & $0.2710 \%$ \\
\hline $\begin{array}{c}\text { Two anchor } \\
\text { nodes }\end{array}$ & $0.3908 \%$ & $0.4025 \%$ & $0.2976 \%$ \\
\hline
\end{tabular}

TABLE I is the error statistics of the experiment. In this paper, after 50 experiments, we can draw a conclusion that although the Bonding-box algorithm improved in the paper [13] increased the degree of identification, in the case of the two anchor nodes, the locating error will be larger than the initial Bonding-box algorithm. It is found that when RB-box algorithm compared with the initial Bonding-box algorithm, the entire network locating precision increased by $1.5 \%$ on average, two anchor nodes locating precision on average by $13.6 \%$. This is the simulating results in the more commonly using of wireless sensor networks. If the anchor nodes in less quantities, the effect of RB-box algorithm will be more than other improved algorithms. Or in the case of a more precise localization, the identification of RB-box algorithm is higher. Due to how less will the anchor nodes be, or how precise the localization to be is based on the actual circumstances, this paper does not give the experimental results.

Among the whole wireless sensor network, we use RB-box algorithm to locate only in the case of two anchor nodes, while the case of the two anchor node rarely occurs for the whole wireless sensor network. Therefore, it is negligible for the increasing of the complexity of the improved algorithm for a small part, which is described in the Introduction above. So that power consumption will not be further reduced.

\section{IN CONCLUSION}

At present, the Bounding-box and its improved locating algorithms generally use a square as the node communicating range model of wireless sensor network, which is inconsistent with the actual model and results in a large WSN locating error; and the error is particularly large in the case of two anchor nodes.

The above algorithms have not improved the locating accuracy in the condition of two anchor nodes. Over this problem, this paper proposes a special locating algorithm for two anchor nodes. Combined with the experimental simulation analysis, we can know that: the RB-box algorithm in the two anchor nodes can further reduce the locating errors and improve the degree of identification. The algorithm can be used in all the improved algorithms of Bonding-box algorithm to solve the problem of not being more precise localization in the case of two anchor nodes and to increase the degree of identification of all improved algorithms. Thereby, the RB-box algorithm can further reduce the locating error of all the improved algorithms.

\section{ACKNOWLEDGMENTS}

The authors are thankful to MCAE2016 for the support to develop this document.

The author would like to acknowledge the support by the National Natural Science Foundation of China.

I would like to express my gratitude to all those who have helped me during the writing of this thesis. I gratefully acknowledge the help of my supervisor Professor Caixia Liu. I do appreciate her patience, encouragement, and professional instructions during my thesis writing.

\section{REFERENCES}

[1] Slobodan N.Simic,Shankar Sastry. Distributed Localization in Wireless Ad Hoc Networks, EPRI, EPRI-35352-6089, December, 2001.

[2] Edgar H C. Wireless Sensor Networks: Architectures and Protocols. Boca Raton, Florida: CRC Press LLC, 2004.

[3] EUNCHAN K,KISE0N K.Distance estimation with weighted lease squares for mobile beacon. based localization in wireless sensor networks. IEEE Signal Processing Letters, 2010, 17(6): 559-562.

[4] CHEN Weike, LI Wen Feng, YUAN Bing. Journal of Wuhan University of Technology ,2006,30(2):265-268.

[5] GUO Haiqi.Research of the localization method based on ZigBee.Chengdu: Southwest Communication University, 2007: 19-32.

[6] WANG Dan.Research on the localization method based on RSSI for wireless sensor network.Harbin: Harbin Institute of Technology. 2011: 44-48.

[7] YAO Yingbiao, ZENG Rong, YI Zhiqiang. Bounding box based distributed search localization algorithm for WSN.Journal of Communications, 2012, 33(Z2): 135-140.

[8] LUO Qinghua, PENG Yu, PENG Xiyuan, et a1. A Bounding-box WSN localization method based on outer tangent line. Journal of Harbin Engineering University, 2015, 36(4): 567-572.

[9] SANABRIA-RUSSO L, CANO C, BELLALTA B. Localization procedure for randomly deployed WSNs based on the composability of position estimation protocols//Proceedings of 9th International Wireless Communications and Mobile Computing Conference. Sardinia, Italy, 2013: 621-626.

[10] YANG Yuan, ZHAO Yubin, KYAS M. weighted least-squares by bounding-box(B-WLS)for NLOS mitigation of indoor localization//Proceedings of IEEE Vehicular Technology Conference. Yangzhou, China, 20l3: 1110-l113.

[11] CHENG Wei,SHI Haoshan,WANG Qingwen. Weighted centroid localization algorithm based on different correlation for sensor network. Journal of System Simulation, 2012, 24(2): 389-393.

[12] SHI Xin, ZHANG Linghua. High-precision weighted Bounding Box localization algorithm for wireless sensor network//Proceedings of 2013 IEEE 3rd International Conference on Information Science and Technology (ICIST 2013). Yangzhou, China, 2013: 1110-1113.

[13] LI Wenchen,ZHANG Lei. Research on Weighted Centroid Location Algorithm for Wireless Sensor Network . Computer Simulation, 2013,30(2):191-194.

[14] ZHAO Guangxi. Research on Localization Algorithm for Wireless Sensor Network. Shenyang: Northeastern University, 2008. 